This material is presented to ensure timely dissemination of scholarly and technical work. Copyright and all rights therein are retained by authors

or by other copyright holders. All persons copying this information are expected to adhere to the terms and constraints invoked by each author's copyright.

In most cases, these works may not be reposted without the explicit permission of the copyright holder." IEEE Conference Proceedings - ISSN: 1098-7584

\title{
A practical inference method with several implicative gradual rules and a fuzzy input: one and two dimensions
}

\author{
Hazaël Jones, Didier Dubois, Serge Guillaume and Brigitte Charnomordic
}

\begin{abstract}
A general approach to practical inference with gradual implicative rules and fuzzy inputs is presented. Gradual rules represent constraints restricting outputs of a fuzzy system for each input. They are tailored for interpolative reasoning. Our approach to inference relies on the use of inferential independence. It is based on fuzzy output computation under an interval-valued input. A double decomposition of fuzzy inputs is done in terms of $\alpha$-cuts and in terms of a partitioning of these cuts according to areas where only a few rules apply. The case of one and two dimensional inputs is considered.
\end{abstract}

\section{INTRODUCTION}

Fuzzy logic, as an interface between symbolic and numeric computations, is well known for its ability to represent uncertainty and imprecision inherent in linguistic concepts.

Historically, fuzzy inference systems were devised to perform a reasoning task based upon expert knowledge yielding a continuous numerical ouput, as needed in fuzzy control. Afterwards, many learning methods were added to enhance numerical performance.

Conjunctive rules [8] as the ones used in the Mamdanistyle fuzzy inference systems, represent joint possible input and output values. They do not really fit an interpretation in terms of logic. In [7], we outlined several advantages of implicative rules with respect to conjunctive rules. For instance, with conjunctive rules, the more rules a rule base has, the more imprecise its output becomes. This fact is usually hidden by defuzzification. Furthermore, the fuzzy output width can bias the result. Gradual implicative rules model constraints restricting output values for each input, and have interesting interpolation properties [6]. They are fully compatible with logic. Among these rules, the most interesting for practical purposes use Goguen implication because of its continuous inference result, and Resher-Gaines implication if a non fuzzy output is needed. Implicative rules are more natural to represent expert knowledge [14] as they model constraints mapping input and output values.

Nevertheless, the practical use of these rules with a fuzzy input is difficult. The aim of this article is to show that under

Hazaël Jones is with INRA and Cemagref, UMR ITAP, UMR ASB, place Pierre Viala 34060 Montpellier Cedex 1 FRANCE (phone: +33 499612070 ; email: jones@ensam.inra.fr).

Didier Dubois is with IRIT Université Paul Sabatier 118 Route de Narbonne 31062 Toulouse Cedex 09 (phone: +33 561556331 ; email: dubois@irit.fr).

Serge Guillaume is with Cemagref UMR ITAP 361, rue JF Breton 34196 Montpellier Cedex 5 FRANCE (phone: +33 467046317 ; email: serge.guillaume@montpellier.cemagref.fr).

Brigitte Charnomordic is with INRA, UMR Analyse des Systèmes et Biométrie 2, place Pierre Viala 34060 Montpellier Cedex 1 FRANCE (phone: +33 499612416 ; email: bch@ensam.inra.fr). some condition on input partitions, inference becomes easy due to a double decomposition: by $\alpha$-cut and by partitioning.

In the sequel of this article, section II recalls some rule features. The inference mechanism is discussed in section III. In section IV, we present sufficient conditions to obtain inferential independence. Then, in section $\mathrm{V}$, we propose a decomposition method based on inferential independence that allows to simplify the inference mechanism for rectangular inputs and a limited number of fuzzy sets in the input partition, as explained in section VI. Section VII adresses the two dimensional case.

\section{IMPLICATIVE RULES}

The proposition " $x \in A$ is possible" means $\forall u \in$ $U, \delta_{X}(u) \geq \mu_{A}(u)$ where $\delta_{X}(u)$ is a guaranteed possibility distribution [3] and $U$ is the universe. In contrast, the proposition " $x \in A$ is certain" means:

$$
\forall u \in U, \pi_{X}(u) \leq \mu_{A}(u)
$$

where $\pi_{X}(u)$ is a (potential) possibility distribution.

Conjunctive rules [8] stand for examples of what is possible under various input conditions. On the contrary, with implicative rules, every piece of knowledge is considered as a restriction of possible worlds. They are a direct application of Zadeh's theory on approximate reasoning[15] and are an extension of classical logic.

The generalised modus ponens is of the form $A^{\prime} \wedge(A \rightarrow O) \models O^{\prime}$, where $\models$ is the logical inference, which means that in presence of an approximate fact $A^{\prime}$ and the implication $A \rightarrow O$, we are able to calculate $O^{\prime}$ defined by :

$$
\mu_{O^{\prime}}(v)=\sup _{u \in U} \mu_{A^{\prime}}(u) \top\left(\mu_{A}(u) \rightarrow \mu_{O}(v)\right)
$$

The output $O^{\prime}$ constrains the value of the output variable. When an operator $\rightarrow$ (implication) is obtained from $T$ (conjunction) by residuation, the standard modus ponens $A \wedge(A \rightarrow O) \vDash O$ is recovered for fuzzy rules. Rule aggregation is conjunctive because the possibility in the sense of (1) is potential: a value estimated as possible by a rule can be forbidden by other rules.

There are different kinds of implicative rules: certainty rules and gradual rules. In this article, we only focus on gradual rules. The behavior of gradual implicative rules, "the more $X$ is $A$, then the more $Z$ is $O$ ", depends on the selected implication. We consider in this paper the following implications : 
- Resher-Gaines: $a \rightarrow b=\left\{\begin{array}{cc}1 & \text { if } a \leq b \\ 0 & \text { otherwise }\end{array}\right.$

- Gödel: $a \rightarrow b=\left\{\begin{array}{cc}1 & \text { if } a \leq b \\ b & \text { otherwise }\end{array}\right.$

- Goguen: $a \rightarrow b= \begin{cases}\min (1, b / a) & \text { if } a \neq 0 \\ 1 & \text { otherwise }\end{cases}$

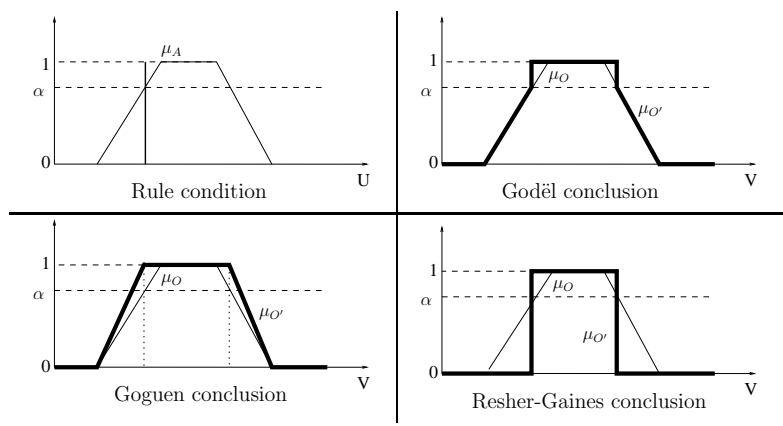

Fig. 1. Inference with a gradual rule and a precise input

\section{INFERENCE MECHANISM}

With implicative rules, the output $O^{\prime}$ is given by:

$$
O^{\prime}=A^{\prime} o \bigcap_{i=1}^{n}\left(A_{i} \rightarrow O_{i}\right)
$$

where $o$ is the composition operator sup-min. When $A^{\prime}$ is a precise input, operators $o$ and $\bigcap$ commute, the output can then be written:

$$
O^{\prime}=\bigcap_{i=1}^{n}\left(A^{\prime} o\left(A_{i} \rightarrow O_{i}\right)\right)
$$

This formalisation corresponds to the FITA ${ }^{1}$ method. This method is easy to implement because the inference can be performed rule by rule.

However, when the input $A^{\prime}$ is imprecise or fuzzy, the commutation of operators $o$ and $\bigcap$ is no longer possible [9]. Only the expression (3) which is a FATI ${ }^{2}$ inference is correct. Currently there is no practical way of handling inference with implicative fuzzy rules, other than the Resher-Gaines case [13]. Another method had been developed in [1] for Gödel implication but the partition fuzzy sets must have overlapping kernels.

For a one dimensional approximate fact, the following inclusion is true:

$$
A^{\prime} o\left(\bigcap_{i=1}^{n} A_{i} \rightarrow O_{i}\right) \subseteq \bigcap_{i=1}^{n}\left(A^{\prime} \circ\left(A_{i} \rightarrow O_{i}\right)\right)
$$

The FITA method only gives an upper approximation of the result.

${ }^{1}$ FITA means "First Infer Then Aggregate"

${ }^{2}$ FATI means "First Aggregate Then Infer"

\section{INFERENTIAL INDEPENDENCE}

To design a practical algorithm for implicative inference, we use the interesting property of inferential independence [12], leading to well-conditioned systems.

\section{A. Definitions and results}

A rule system $\left\{A_{j} \rightarrow O_{j}, j=1 \ldots, n\right\}$ is wellconditioned if it produces the output fact $O_{i}$ when fed with the input fact $A_{i}$, for any $i=1 \ldots, n$ :

$$
\forall i, A_{i} O \bigcap_{j}\left(A_{j} \rightarrow O_{j}\right)=O_{i}
$$

The partition shape will determine if the system is wellconditioned or not. More often than not, this equation is not true, and is replaced by the following assertion:

$$
A_{i} O \bigcap_{j}\left(A_{j} \rightarrow O_{j}\right)=O_{i}^{\prime}
$$

where $O_{i}^{\prime} \subset O_{i}$. According to Morsi[11], if we substitute each output of a system by the inferred output $O_{j}^{\prime}$, the system $A_{j} \rightarrow O_{j}^{\prime}$ is well-conditioned:

$$
A_{i} O \bigcap_{j}\left(A_{j} \rightarrow O_{j}^{\prime}\right)=O_{i}^{\prime}
$$

Morsi's proof uses residuated implication properties [10] verified by Gödel and Goguen operators and the relation: $\bigcap_{j}\left(A_{j} \rightarrow O_{j}\right)=\bigcap_{j}\left(A_{j} \rightarrow O_{j}^{\prime}\right)$ proved in [11]. In a well-conditioned system, rules are inferentially independent.

In the sequel, we check which types of partition yield a well-conditioned system. The following proof does not work for Resher-Gaines but it is true for all residuated implications obtained from a continuous t-norm.

\section{B. Sufficient conditions}

From equation (2), and because of the conjunctive aggregation of implicative rules, we obtain with the min conjunction operator: $\forall y \in V$,

$$
\sup _{x \in U} \mu_{A_{i}}(x) \top \min _{j \in N}\left(\mu_{A_{j}}(x) \rightarrow \mu_{O_{j}}(y)\right)=\mu_{O_{i}}(y)
$$

By shifting $\mu_{A_{i}}(x)$ and t-norm $T$ inside of min: $\forall y$,

$$
\sup _{x \in U} \min _{j \in N}\left(\mu_{A_{i}}(x) \top\left(\mu_{A_{j}}(x) \rightarrow \mu_{O_{j}}(y)\right)\right)=\mu_{O_{i}}(y)
$$

We are looking for sufficient conditions for this equality to hold. Since $O_{i}^{\prime} \subseteq O_{i}$, the equation is equivalent to: $\forall y, \exists x \in$ $U$,

$$
\min _{j \in N}\left(\mu_{A_{i}}(x) \top\left(\mu_{A_{j}}(x) \rightarrow \mu_{O_{j}}(y)\right)\right)=\mu_{O_{i}}(y)
$$

Then, we have the following sufficient conditions: $\forall y, \exists x \in U$,

$$
\text { nd } \begin{array}{r}
\forall j \neq i, \mu_{A_{i}}(x) \top\left(\mu_{A_{j}}(x) \rightarrow \mu_{O_{j}}(y)\right) \geq \mu_{O_{i}}(y) \\
\mu_{A_{i}}(x) \top\left(\mu_{A_{i}}(x) \rightarrow \mu_{O_{i}}(y)\right)=\mu_{O_{i}}(y)
\end{array}
$$


Choosing $x \in \operatorname{Core}\left(A_{i}\right)$, equation (5) obviously holds since $1 \rightarrow \mu_{O_{i}}(y)=\mu_{O_{i}}(y)$ for Gödel and Goguen implication. For Resher-Gaines implication, this equation holds if, $\forall y$, we choose $x$ such that $\mu_{A_{i}}(x)=\mu_{O_{i}}(y)$.

Now, we must check equation (4). If we consider $x$ in the core of $A_{i}$, then a sufficient condition is the following: $\forall y, \exists x \in \operatorname{Core}\left(A_{i}\right), \forall j \neq i$,

$$
\mu_{A_{j}}(x) \rightarrow \mu_{O_{j}}(y) \geq \mu_{O_{i}}(y)
$$

because $1 \top x=x$. Then we have two cases:

- $\mu_{A_{j}}(x)>\mu_{O_{j}}(y)$ : then equation (6) is not usually true. If this strict inequality holds for all $x \in \operatorname{Core}\left(A_{i}\right)$, the system is not well-conditioned.

- $\mu_{A_{j}}(x) \leq \mu_{O_{j}}(y)$ : then equation (6) is always true.

In order to respect this condition, partitions must satisfy the following property: at least one value in a fuzzy set core must not belong to the support of other sets. i.e. as we can see on figure $2, \exists x \in \operatorname{Core}\left(A_{i}\right), \mu_{A_{j}}(x)=0, \forall j \neq i$.

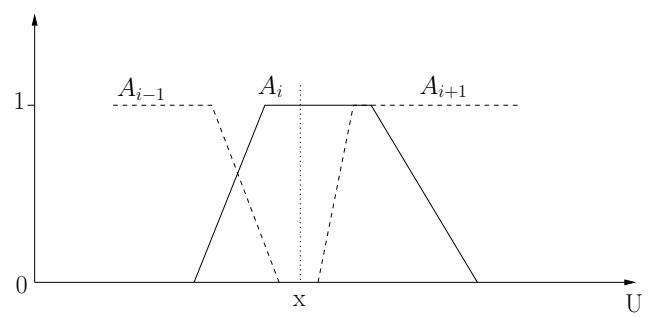

Fig. 2. A fuzzy partition allowing inferential independance

For strong input fuzzy partitions (see figure 4) the following stronger property holds: $\forall j \neq i, \forall x \in \operatorname{Core}\left(A_{i}\right)$, $\mu_{A_{j}}(x)=0$. Hence the system is always well-conditioned in this case.

We now obtain an interesting property useful for inference: for strong fuzzy partitions, with $x \in \operatorname{Core}\left(A_{i}\right)$, the system output is $O_{i}$ for Gödel and Goguen and $\operatorname{Core}\left(O_{i}\right)$ for Resher-Gaines. This proof holds for a n-dimensional system.

\section{DECOMPOSITION BASED ALGORITHM}

Now, we use strong fuzzy partitions and the inferential independence property to design a practical inference process by input decompositions. These decompositions are feasible due to the following property of a fuzzy relation $R$ :

$$
\left(A \cup A^{\prime}\right) o R=(A o R) \cup\left(A^{\prime} \circ R\right)
$$

First we consider one-dimensional inputs.

\section{A. $\alpha$-cut decomposition}

An $\alpha$-cut of $A$ is an interval defined by: $\forall \alpha>0, I_{A_{\alpha}}=\left\{x \in \mathbb{R} \mid \mu_{A}(x) \geq \alpha\right\}=\left[I_{\alpha l}, I_{\alpha r}\right]$

$A$ is equal to $\bigcup_{\left.\left.\alpha_{j} \in\right] 0,1\right]} A_{\alpha_{j}} \times \alpha_{j}$ where $A_{\alpha_{j}}$ is a rectangular input of level 1 whose width is the $\alpha$-cut $I_{A_{\alpha_{j}}}$. A fuzzy set $A$ is bracketted within two approximations: inner and external (see figure 3).

$$
\bigcup_{j=1, \ldots, n} \alpha_{j} A_{\alpha_{j}} \subseteq A \subseteq \bigcup_{j=1, \ldots, n} \alpha_{j} A_{\alpha_{j+1}}
$$

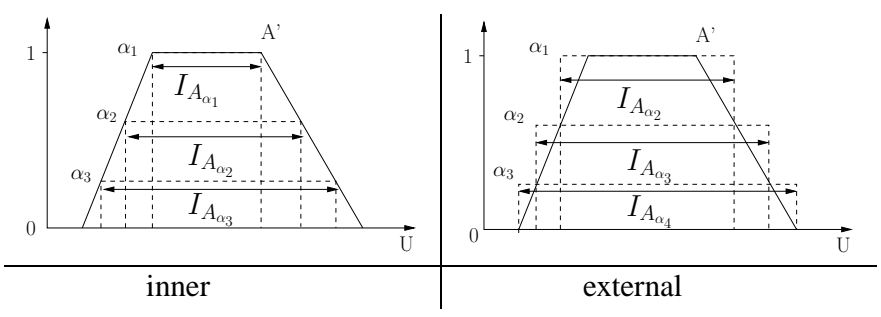

Fig. 3. $\alpha$-cut decomposition

External approximations seem to be more appropriate because they include the fuzzy input. The approximated output will also contain the true output. It could be interesting to keep both inner and external approximations in order to reason with two approximations like for Rough Sets [2].

Equation (8) shows that the number of $\alpha$-cut chosen will influence the inferred output accuracy.

\section{B. Partitioning decomposition}

To partition the input space, we consider supports and cores seperately. Let $E_{k}$ be intervals obtained from such a decomposition (see figure 4). This decomposition is able to isolate the fuzzy set cores. Thanks to the well-conditioned system property, inference is straightforward in the core area.

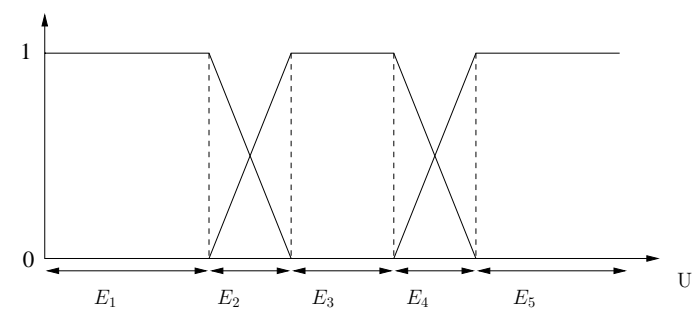

Fig. 4. Partitioning decomposition with strong fuzzy partition

In the presence of a fuzzy input $A^{\prime}$ on an input partition, we first decompose $A^{\prime}$ using an $\alpha$-cut decomposition. Then, the partitioning decomposition will be carried out on $A^{\prime}$ 's support. As a consequence, we have the relation:

$$
A^{\prime}=\bigcup_{\alpha}\left(\alpha\left(\bigcup_{k=1, \ldots, p} E_{k} \cap A_{\alpha}\right)\right)
$$

where $p$ is the number of intervals $E_{k}$.

\section{INFERENCE WITH A RECTANGULAR INPUT}

Output $O^{\prime}$ is then equal to:

$$
O^{\prime}=\bigcup_{\alpha}\left(\alpha \top\left(\bigcup_{k=1, \ldots, p} O_{k}^{\prime}\right)\right)
$$

where $O_{k}^{\prime}=\left(E_{k} \cap A_{\alpha}\right) o R$ and $\top$ the $\mathrm{t}$-norm of the residuated implication. Due to partitioning, there are at most two fuzzy sets by decomposition. Furthermore, due to $\alpha$-cut decomposition, it all comes down to handling rectangular inputs. If the rectangular input is included within the fuzzy 


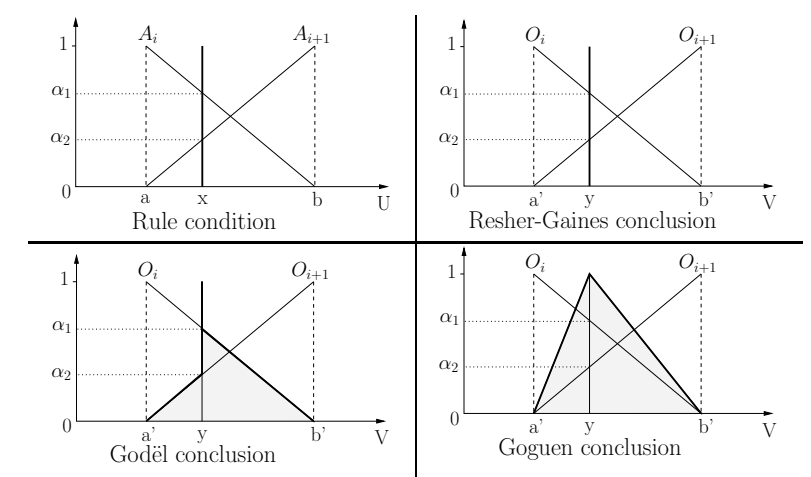

Fig. 5. Inference with two gradual implicative rules and a precise input

set core of $A_{i}$, the result is obvious: we obtain $O_{i}$ for Gödel and Goguen and $O_{i}$ 's core for Resher-Gaines.

Let the interval of interest be $\left[i_{l}, i_{r}\right]$. A rectangular input membership function is defined by $\mu_{\left[i_{l}, i_{r}\right]}$ such that: $\mu_{\left[i_{l}, i_{r}\right]}(x)= \begin{cases}\alpha & \text { if } i_{l} \leq x \leq i_{r} \\ 0 & \text { otherwise }\end{cases}$

If a rectangular input is included within the overlapping of two fuzzy sets (see figure 6), the output is given by:

$$
\mu_{O^{\prime}}(y)=\sup _{a \leq x \leq b} \min _{i=1, \ldots, n}\left(\mu_{\left[i_{l}, i_{r}\right]}(x) \top \mu_{A_{i}}(x) \rightarrow \mu_{O_{i}}(y)\right)
$$

In this specific case, it is equal to:

$$
\begin{aligned}
\mu_{O^{\prime}}(y)= & \sup _{a \leq x \leq b} \min \left(\mu_{\left[i_{l}, i_{r}\right]}(x) \top \mu_{A_{i}}(x) \rightarrow \mu_{O_{i}}(y),\right. \\
& \left.\mu_{\left[i_{l}, i_{r}\right]}(x) \top \mu_{A_{i+1}}(x) \rightarrow \mu_{O_{i+1}}(y)\right)
\end{aligned}
$$

for some $i$. As the input is rectangular, there are two cases:

- Let $\mu_{\left[i_{l}, i_{r}\right]}(x)=0$, then we can deduce that $\mu_{O^{\prime}}(y)=0$ because $0 \top x=0$.

- Let $\mu_{\left[i_{l}, i_{r}\right]}(x)=\alpha$, then we get the following equation:

$$
\begin{aligned}
\mu_{O^{\prime}}(y)= & \sup _{i_{l} \leq x \leq i_{r}} \min \left(\alpha \top \mu_{A_{i}}(x) \rightarrow \mu_{O_{i}}(y),\right. \\
& \left.\alpha \top \mu_{A_{i+1}}(x) \rightarrow \mu_{O_{i+1}}(y)\right)
\end{aligned}
$$

Since $\alpha$ and $\top$ are independent of $x$ and $i$, the system is equivalent to:

$$
\begin{gathered}
\mu_{O^{\prime}}(y)=\alpha \top \sup _{i_{l} \leq x \leq i_{r}} \min \left(\mu_{A_{i}}(x) \rightarrow \mu_{O_{i}}(y),\right. \\
\left.\mu_{A_{i+1}}(x) \rightarrow \mu_{O_{i+1}}(y)\right)
\end{gathered}
$$

Next, the output's behavior depends on the chosen residuated implication. We'll consider Resher-Gaines, Gödel and Goguen implication. Figure 5 displays inference results with a crisp input and two gradual rules.

Level $\alpha$ has only a limiting effect on the output's height. No output element could have higher membership than level

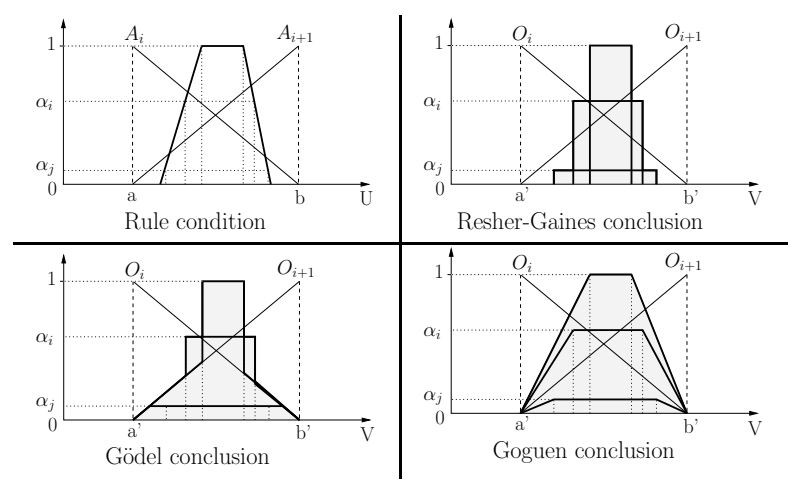

Fig. 6. Inference with two gradual implicative rules and a fuzzy input decomposed on three levels $\alpha_{j}<\alpha_{i}<1$

$\alpha$ because the minimum is the upper bound of t-norms. According to the chosen implication, a different t-norm will be used. For Resher-Gaines and Gödel, t-norm is the minimum. Then, the output is truncated at level $\alpha$, but its shape is preserved. For Goguen implication, t-norm is the product. The output is also truncated at level $\alpha$ but the support slopes are modified (See figure 6).

Output computation for one rectangular input is straightforward depending on the chosen implication. The onedimensional inference process is done by performing the union of outputs inferred from each rectangular input taking both decomposition into account.

\section{2D INFERENCE}

In this section, we focus on Resher-Gaines implication because the computation provides the core of outputs inferred using residuated fuzzy implications. We need to make sure fuzzy rule conclusions ensure logical system coherence [5]. Then several cases must be studied according to input location.

\section{A. Fuzzy rule conclusions and coherence}

As a precise input value may belong to at most two fuzzy sets in each dimension $\left(A_{1}\right.$ and $A_{2}, B_{1}$ and $\left.B_{2}\right)$, four rules are likely to be simultaneously triggered by a $2 \mathrm{D}$ precise input:

- If $X$ is $A_{1}$ and $Y$ is $B_{1}$ then $Z$ is $O_{1,1}$

- If $X$ is $A_{1}$ and $Y$ is $B_{2}$ then $Z$ is $O_{1,2}$

- If $X$ is $A_{2}$ and $Y$ is $B_{1}$ then $Z$ is $O_{2,1}$

- If $X$ is $A_{2}$ and $Y$ is $B_{2}$ then $Z$ is $O_{2,2}$

Contrary to the one-dimensional case where rule incoherence is easily spotted, the two-dimensional rule system is not so easy to deal with regarding coherence. In order to maintain system coherence, we must have $O_{1,1} \cap O_{1,2} \cap O_{2,1} \cap O_{2,2} \neq \emptyset$. To insure coherence [4], we build an output coverage (see figure 8) given input partitions (see figure 7) and a monotonic continuous 2D function. We build outputs such as $O_{1,1}=f\left(A_{1}, B_{1}\right), O_{1,2}=f\left(A_{1}, B_{2}\right), O_{2,1}=f\left(A_{2}, B_{1}\right)$ and $O_{2,2}=f\left(A_{2}, B_{2}\right)$. 

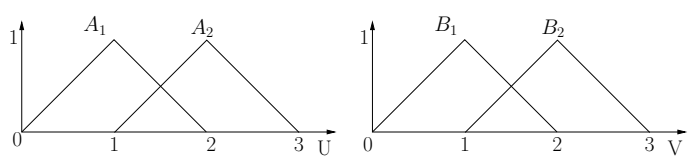

Fig. 7. Input partitions

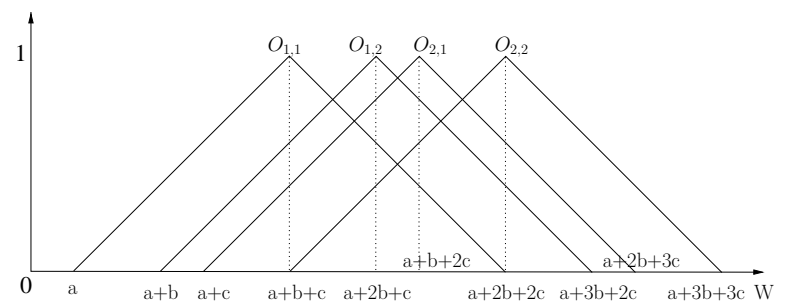

Fig. 8. Output partition created with a linear function $a+b x+c y$

As an example, a linear function $f(x, y)=a+b x+c y$, creates an output partition calculated from input vertices as in figure 8. In this figure, $O_{1,1}$ and $O_{2,2}$ still make a strong partition. If $b=c$, then $O_{1,2}$ and $O_{2,1}$ are identical.

\section{B. 2D inference difficulties}

With input strong partitions, there are 3 different situations according to the precise input location (see figure 9). In the sequel, we denote $a^{-}(\alpha)$ the smaller of the two abcissas of $U$ whose membership degree to $A$ is $\alpha$. The greater value of the two abcissas is denoted $a^{+}(\alpha)$ (see figure 10).

- Case 1: both inputs lie within the cores of fuzzy sets $A_{k}$ in $U$ and $B_{l}$ in $V$. In this situation we can directly infer the output thanks to the rule inferential independence (see section IV). Output is equal to $\operatorname{Core}\left(O_{k, l}\right)$ for Resher-Gaines implication.

- Case 2: the $x$ input lies within the core of a fuzzy set $A_{k}$ and the $y$ input lies between the cores of $B_{l}$ and $B_{m}$. In

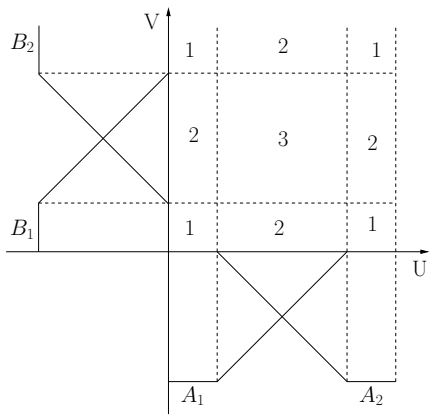

Fig. 9. Areas defined by input partitions

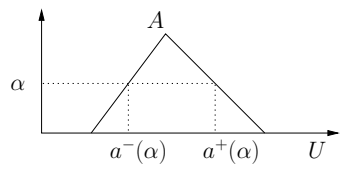

Fig. 10. Input notation

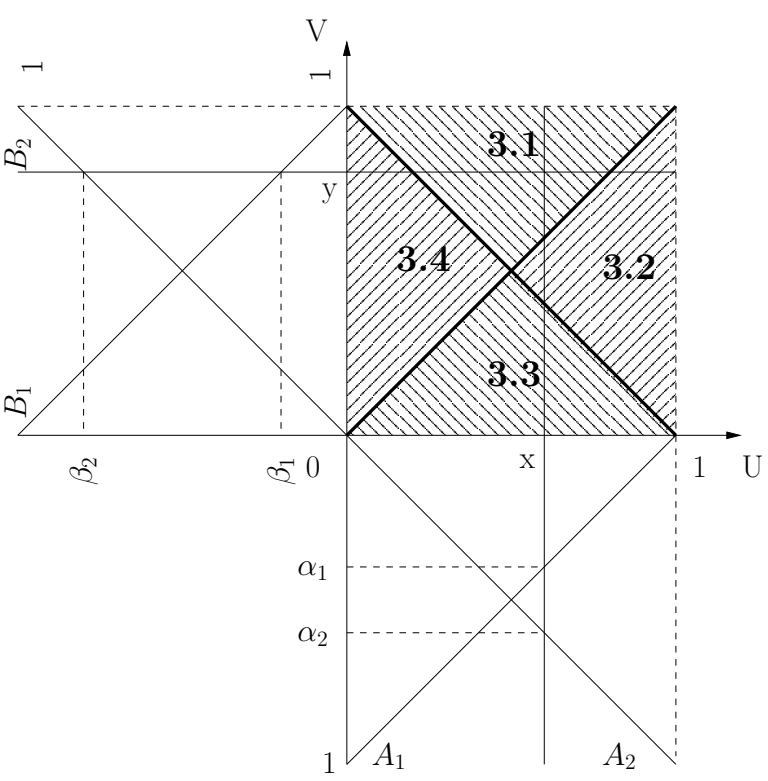

Fig. 11. Case 3: four interesting areas

consequence, only 2 rules are triggered: $A_{k} \wedge B_{l} \rightarrow O_{k, l}$ and $A_{k} \wedge B_{m} \rightarrow O_{k, m}$. This behavior is the same for an input $y$ within the core of $B_{k}$ and an input $x$ between $A_{l}$ and $A_{m}$ 's cores.

- Case 3: the input lies between the cores of adjacent fuzzy sets in $U$ and in $V$ (see figure 11). Four rules are triggered. This is the most complicated case.

Given a function $f(x, y)$ and a 2D precise input, we can compute the Resher-Gaines output [4], which is an interval: expressions of the lower bound $z_{\min }$ and upper bound $z_{\max }$ are summed up in table I for each zone (see figure 11). Zones are defined according to the value of $m=\min \left(\alpha_{1}, \alpha_{2}, \beta_{1}, \beta_{2}\right)$ (see fig. 7, where $\alpha_{1}=1-$ $\left.\alpha_{2}, \beta_{1}=1-\beta_{2}\right)$ : if $m=\beta_{1}$ we are in zone 3.1, $m=\alpha_{1}$ corresponds to zone $3.2, m=\beta_{2}$ to zone 3.3 and $m=\alpha_{2}$ to zone 3.4.

\section{Continuity and kink points with rectangular inputs}

In presence of a rectangular input, it is important to know if we can compute output upper and lower bounds using only the vertices of the input. We need to know if the real output is in the convex hull of outputs inferred with the two vertices of the rectangular input.

To illustrate this problem, we consider a precise input on dimension $V$ and we study the output evolution according to the other dimension (see figure 12). This figure shows a continuous output and a kink point in the lower output bound for some value of $\alpha_{1}$. It is important to detect this point to obtain the output convex hull.

A kink point is typically obtained if the two functions defining an output bound (table I) evolve in opposite directions. For example, in figure 12, a kink point appears on the lower bound in zone 3.1. This bound is the result of expression: 


\begin{tabular}{|c|c|}
\hline Zone & Lower bound $z_{\min }$ \\
\hline \hline 3.1 & $\max \left(f\left(a_{2}^{-}\left(\alpha_{2}\right), b_{2}^{-}\left(\alpha_{2}\right)\right), f\left(a_{1}^{-}\left(\alpha_{1}\right), b_{2}^{-}\left(\alpha_{1}\right)\right)\right)$ \\
\hline 3.2 & $\max \left(f\left(a_{2}^{-}\left(\beta_{2}\right), b_{2}^{-}\left(\beta_{2}\right)\right), f\left(a_{2}^{-}\left(\beta_{1}\right), b_{1}^{-}\left(\beta_{1}\right)\right)\right)$ \\
\hline 3.3 & $\max \left(f\left(a_{2}^{-}\left(\alpha_{2}\right), b_{1}^{-}\left(\alpha_{2}\right)\right), f\left(a_{2}^{-}\left(\beta_{2}\right), b_{2}^{-}\left(\beta_{2}\right)\right)\right)$ \\
\hline 3.4 & $\max \left(f\left(a_{2}^{-}\left(\alpha_{2}\right), b_{2}^{-}\left(\alpha_{2}\right)\right), f\left(a_{1}^{-}\left(\beta_{2}\right), b_{2}^{-}\left(\beta_{2}\right)\right)\right)$ \\
\hline \hline Zone & Upper bound $z_{\max }$ \\
\hline 3.1 & $\min \left(f\left(a_{1}^{+}\left(\beta_{1}\right), b_{1}^{+}\left(\beta_{1}\right)\right), f\left(a_{1}^{+}\left(\alpha_{1}\right), b_{2}^{+}\left(\alpha_{1}\right)\right)\right)$ \\
\hline 3.2 & $\min \left(f\left(a_{2}^{+}\left(\beta_{1}\right), b_{1}^{+}\left(\beta_{1}\right)\right), f\left(a_{1}^{+}\left(\alpha_{1}\right), b_{1}^{+}\left(\alpha_{1}\right)\right)\right)$ \\
\hline 3.3 & $\min \left(f\left(a_{2}^{+}\left(\alpha_{2}\right), b_{1}^{+}\left(\alpha_{2}\right)\right), f\left(a_{1}^{+}\left(\alpha_{1}\right), b_{1}^{+}\left(\alpha_{1}\right)\right)\right)$ \\
\hline 3.4 & $\min \left(f\left(a_{1}^{+}\left(\beta_{1}\right), b_{1}^{+}\left(\beta_{1}\right)\right), f\left(a_{1}^{+}\left(\beta_{2}\right), b_{2}^{+}\left(\beta_{2}\right)\right)\right)$ \\
\hline
\end{tabular}

TABLE I

OUtPUt InTERVALS $\left(\alpha_{1}=\alpha_{2}-1, \beta_{1}=\beta_{2}-1\right)$

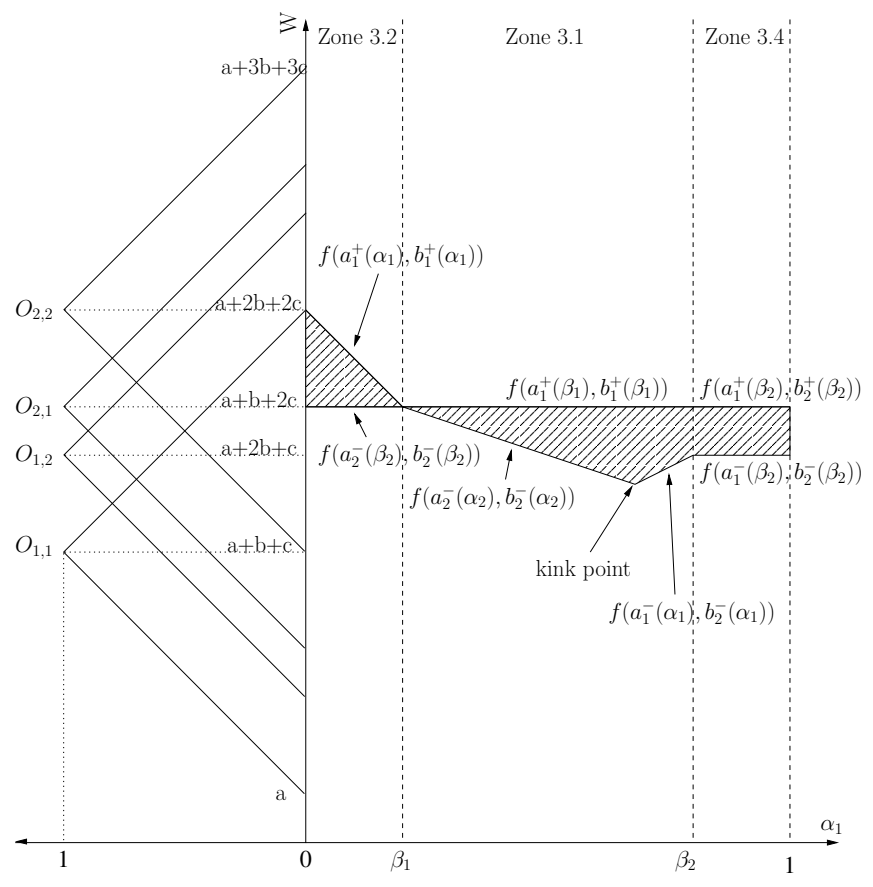

Fig. 12. Output evolution according to $\alpha_{1}$

$\max \left(f\left(a_{2}^{-}\left(\alpha_{2}\right), b_{2}^{-}\left(\alpha_{2}\right)\right), f\left(a_{1}^{-}\left(\alpha_{1}\right), b_{2}^{-}\left(\alpha_{1}\right)\right)\right)$,

where the former decreases and the latter increases. Consequently, the kink point is where $f\left(a_{2}^{-}\left(\alpha_{2}\right), b_{2}^{-}\left(\alpha_{2}\right)\right)=f\left(a_{1}^{-}\left(\alpha_{1}\right), b_{2}^{-}\left(\alpha_{1}\right)\right)$. For a linear function $f(x, y)=a+b x+c y$, this equality becomes: $a+a_{2}^{-}\left(\alpha_{2}\right) b+b_{2}^{-}\left(\alpha_{2}\right) c=a+a_{1}^{-}\left(\alpha_{1}\right) b+b_{2}^{-}\left(\alpha_{1}\right) c$. All terms of this equation are known so we can find the membership level $\alpha_{1}$ that corresponds to the kink point.

To sum up the inference process in two dimensions with rectangular inputs, it is necessary to:

- infer from each vertex of the rectangular input.
- test if there are kink points those input values are within the rectangular input.

- infer from all input values yielding kink points.

The final output is the convex hull of all the outputs so inferred.

\section{CONCLUSION}

This paper lays the foundation for a practical inference method with a system of implicative fuzzy rules. In the 2D case the difficult point is to infer from a rectangular (imprecise) input. For fuzzy input, we can get an approximation of the result using $\alpha$-cuts and partitioning decomposition. Inferring with this kind of fuzzy system is especially appropriate when modeling expert knowledge expressing constraints (as opposed to Mamdani rules). In the future, this method will be tested on a predictive diagnosis case-study of cheese production process, for which expert rules with two input conditions are frequent. Extending the approach beyond 2D inputs is also the next challenging task.

\section{REFERENCES}

[1] Didier Dubois, Roger Martin-Clouaire, and Henri Prade. Practical computing in fuzzy logic, pages 11-34. Fuzzy Computing, 1988.

[2] Didier Dubois and Henri Prade. Rough fuzzy sets and fuzzy rough sets. International Journal of General Systems, 17:191-209, 1990.

[3] Didier Dubois and Henri Prade. What are fuzzy rules and how to use them. Fuzzy Sets and Systems, 84(2):169-185, 1996.

[4] Didier Dubois, Henri Prade, and Michel Grabisch. Gradual rules and the approximation of control laws. Theoretical aspects of fuzzy control, pages 147-181, 1995.

[5] Didier Dubois, Henri Prade, and Laurent Ughetto. Checking the coherence and redundancy of fuzzy knowledge bases. IEEE Transactions on Fuzzy Systems, 5:398-417, 1997.

[6] Sylvie Galichet, Didier Dubois, and Henri Prade. Imprecise specification of ill-known functions using gradual rules. Int. J. Approx. Reasoning, 35(3):205-222, 2004.

[7] Hazaël Jones, Serge Guillaume, Brigitte Charnomordic, and Didier Dubois. Practical use of fuzzy implicative gradual rules in knowledge representation and comparison with mamdani rules. EUSFLAT, 2005.

[8] E H Mamdani and S Assilian. An experiment in linguistic synthesis with a fuzzy logic controller. International journal on man-machine studies, 7:1-13, 1975.

[9] R Martin-Clouaire. Semantics and computation of the generalized modus ponens: The long paper. International journal of Approximate Reasoning, 3:195-217, 1987.

[10] Nehad N. Morsi. A small set of axioms for residuated logic. Inf. Sci., 175(1-2):85-96, 2005.

[11] Nehad N. Morsi and Aly A. Fahmy. On generalized modus ponens with multiple rules and a residuated implication. Fuzzy Sets Syst., 129(2):267-274, 2002.

[12] Laurent Ughetto. Inferential independence of fuzzy rules. In ECAI, pages 605-609, 1998.

[13] Laurent Ughetto, Didier Dubois, and Henri Prade. Efficient inference procedures with fuzzy inputs. In FUZZY-IEEE'97, pages 567-572, Barcelona, 1997.

[14] Laurent Ughetto, Didier Dubois, and Henri Prade. Implicative and conjunctive fuzzy rules - A tool for reasoning from knowledge and examples. In AAAI-99, Orlando, Floride (USA), pages 214-219, California, 1999. AAAI Press/The MIT Press.

[15] L A Zadeh. A theory of approximate reasoning. Machine Intelligence, 9:149-194, 1979. 